\title{
A Rehabilitation of Missing Maxillary Anterior Teeth in a Severe Skeletal Class III Malocclusion Patient Requiring Implants
}

www.adum.edu.my

Ann Dent UM 2021, 28: 8-14

Dol: 10.22452/adum.vol28no2

\section{Zethy Hanum Mohamed Kassim ${ }^{1 *}$, Abdul Latif Abdul Hamid ${ }^{2}$, Nadhirah Ghazali ${ }^{1}$, Puvanendran Balasingham ${ }^{3}$}

\section{KEYWORDS}

Class III malocclusion, Decompensation, Orthognathic, Implants, Prosthesis

\begin{abstract}
Management of traumatic dental injuries (TDI) in a young patient may range from simple to complex. In a situation where teeth are lost, a reliable and conservative treatment option is an implant-supported fixed dental prosthesis (i-FDP), as this treatment option negates the need to prepare sound abutment teeth as in the case of conventional fixed bridges. However, the placement of implants is usually prosthetically driven to allow for a 3D functional and aesthetic restoration. In the presence of severe skeletal Class III malocclusion, treatment may incorporate pre-surgical orthodontic treatment, followed by jaw surgery to correct the skeletal discrepancies and finally post-surgical orthodontic treatment before the rehabilitation with implants. A multidisciplinary treatment approach in a stepwise manner is required to address the patient's overall treatment needs. This case report presents a joint prosthodontics, orthodontics and oral maxillofacial surgical management of a young adult male patient with a Skeletal Class III malocclusion who required rehabilitation of avulsed missing anterior teeth sustained from childhood TDI. The severity of the skeletal relationship required a Le Fort I maxillary advancement and a bilateral sagittal split osteotomy for the setback of the mandible in combination with orthodontics for correction of malocclusion and arch relationship prior to implant placement. Correction of the malocclusion and jaw deformity allowed the functional and aesthetic rehabilitation of the missing teeth using an i-FDP.
\end{abstract}

\section{INTRODUCTION}

Traumatic dental injuries cause damage to the structures within the oral cavity that may include the teeth, periodontal apparatus, mucosa, bone and musculature. In children and adolescents, the most common aetiology reported is fall and others are sports, traffic accidents, fall from bicycle/tricycle or indoor and outdoor play. ${ }^{1} \mathrm{~A}$ recent meta-analysis reported that more than 1 billion people had experienced TDI translating to a global prevalence of $15.2 \%$ with an incidence rate

\footnotetext{
${ }^{1}$ Restorative Department, Faculty of Dentistry, Universiti Teknologi MARA (UiTM), Sungai Buloh Campus, Selangor ${ }^{2}$ Columbia Asia Medical Centre, Seremban, Negeri Sembilan ${ }^{3}$ Private Practice, Pristine Dental Centre, Wilayah Persekutuan, Kuala Lumpur

*Correspondence: zethy@uitm.edu.my
}

of 2.75 for permanent teeth and 2.72 for primary teeth per 100 persons per year. ${ }^{2}$ Injuries sustained to permanent teeth in children and adolescents may range from simple fractures to avulsion resulting in different degrees of complexities of the therapeutic need of the patient.

This case report focuses on the sequence of management of a patient who requested for implants for the replacement of avulsed maxillary teeth during childhood that was complicated by the presence of Class III malocclusion on a severe Class III skeletal base. Dental features of this patient included maxillary incisors protrusion and mandibular incisors retrusion with an anterior open bite which is challenging for Prosthodontic rehabilitation solely with implants to develop functional overjet and overbite, anterior guidance, 
overall facial and dental aesthetics. The management that is unique to this Class III patient is the need for correction of his skeletal and dental relationship before the commencement of prosthodontic treatment despite the chief complaint that was prosthodontic related.

Treatment of this patient necessitated a multidisciplinary management team that consisted of a prosthodontist, an orthodontist and an oral surgeon with an aim to correct the Class III malocclusion, skeletal relationship and restore the missing maxillary anterior teeth.

\section{PATIENT INFORMATION}

The present case was conducted according to the 1964 Helsinki declaration and reported in compliance with the CARE guidelines. A 21-yearsold Malay patient came to the Prosthodontic clinic requesting to replace his missing maxillary front teeth with implants as he is not happy with the chrome-cobalt denture that was provided to him by his previous dentist. Apart from this, he complained of difficulty eating, particularly of noodles because he is not able to bite with his front teeth. The patient's teeth were missing due to a fall he experienced during childhood following a bicycle accident. He was otherwise medically fit and healthy. At the time of the initial visit, the patient was a final year student studying quantity surveying at a local university. He had undergone treatment with numerous clinicians since childhood and the treatment received included endodontic treatment of teeth 22 and 23 (Figure 3b). His last dental treatment was the provision of a maxillary chromecobalt denture. He had been informed regarding implants as a treatment option to replace his missing teeth. However, the option of orthognathic surgery prior to implant placement had never been discussed.

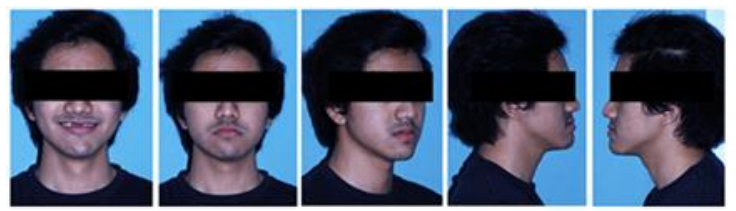

Figure 1: Pre-treatment extraoral view photographs

\section{CLINICAL PRESENTATION}

Extraoral examination revealed a Skeletal Class III pattern with a retrognathic maxilla and prognathic mandible (Figure 1). Intraorally the patient had missing teeth 11,12 and 21 that were replaced with a chrome-cobalt denture at the time of presentation (Figure 2a). With the denture ex-situ, labial bone defect was noted, extending around the region of the missing teeth (Figure $2 b$ ). With the denture in situ, a Class III incisor relationship with a $5 \mathrm{~mm}$ of reverse overjet was measured at the region of the incisor with the presence of an anterior open bite. He also had a full unit Class III canine and molar relationship on the right side and on the left side (Figures $2 \mathrm{c}$ and $2 \mathrm{~d}$ ).
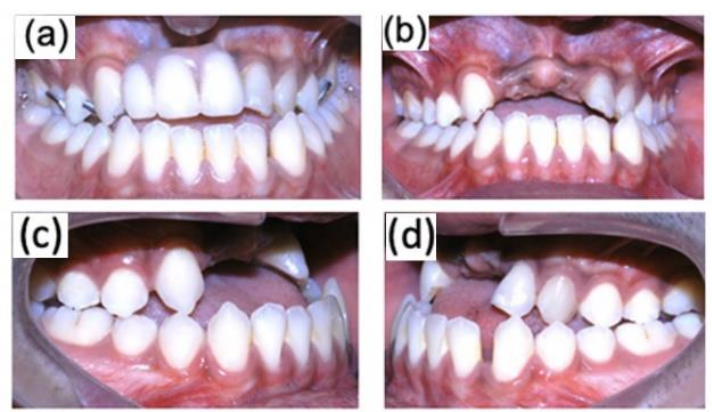

Figure 2: Pre-treatment intraoral photographs with and without maxillary denture in-situ

\section{DIAGNOSTIC ASSESSMENT}

Analysis of the lateral cephalometric radiograph taken (Figure 3a) revealed SNA $=83^{\circ}, \mathrm{SNB}=86^{\circ}$, $\mathrm{ANB}=-1^{0}$, Wit's Analysis $=-17 \mathrm{~mm}$, UInc to $\mathrm{MxPI}=$ $122^{\circ}$, Linc to $\mathrm{MnPI}=85^{\circ}$, Inter-incisal angle $=124^{\circ}$, $\mathrm{MMPA}=31^{\circ}, \mathrm{LAFH}=54 \%$, Linc to APog line $=10 \mathrm{~mm}$, $\mathrm{SN}$ to $\mathrm{MxPI}=11^{0}$.
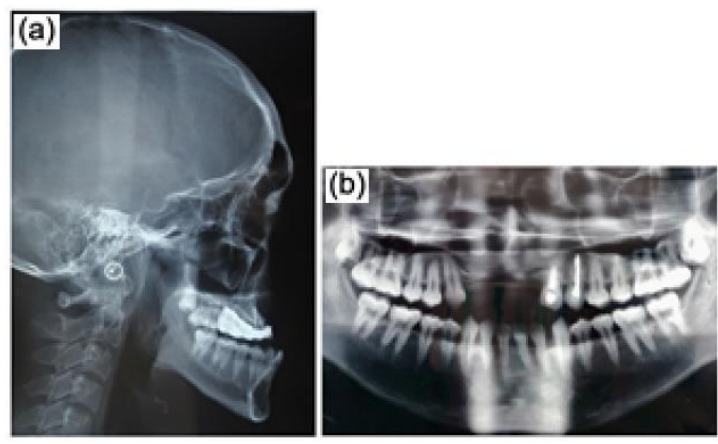

Figure 3: (a) Pre-treatment lateral cephalometric radiograph and (b) Pre-treatment orthopantomogram (OPG).

Pre-orthognathic surgery diagnostic aid included model surgery whereby, cuts for maxillary advancement and mandibular setback were simulated on a set of study casts that were mounted on a semi-adjustable articulator. Acrylic splints were fabricated on these casts and used intra-operatively to obtain the desired position of the maxilla and the mandible prior to fixation with mini-plates and screws. Before the implant surgery, a diagnostic wax-up was made on a set of study casts mounted in intercuspal position (Figure 4a). A duplicate cast of this diagnostic wax-up was used to fabricate the radiographic/surgical stent for 
implant position planning pre-operatively and placement intra-operatively. Maxillary cone-beam computed tomography (CBCT) was taken following completion of post-surgical orthodontic treatment as shown in Figures $4 \mathrm{~b}$ to $4 \mathrm{~d}$.
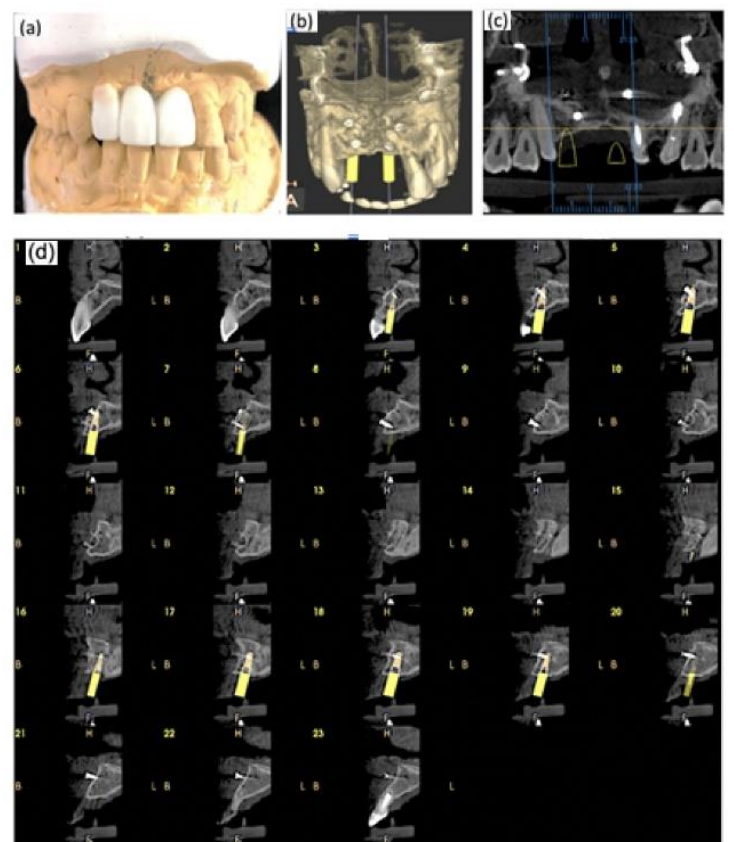

Figure 4: Implant planning (a) Diagnostic wax-up of teeth 12 to 21, (b) Coronal slice of maxillary CBCT with implants at teeth 12 and 21 positions. The image also shows the position of fixation screws following buccal bone grafting, (c) Coronal cut of maxillary $\mathrm{CBCT}$ indicating the area for the sagittal slices, (d) Sagittal slices of maxillary CBCT whereby slices 3 to 7 depict presence of remodelled buccal bone graft and position of implant for replacement of tooth 12 , slices 16 to 20 depict presence of remodelled buccal bone graft and position of implant for replacement of tooth 21 , and slices 13 to 15 depict location of the incisive canal

\section{THERAPEUTIC INTERVENTION}

The patient underwent a period of pre-surgical orthodontic treatment in preparation for the bimaxillary surgery for 24 months. Treatment was aimed at decompensation of the maxillary and mandibular teeth, aligning and levelling of all teeth (Figure 5a). The maxillary archwire was removed in anticipation of the bone grafting planned at the region of missing teeth 11,12 and 21 during the orthognathic surgery (refer to buccal concavity visible in Figure $5 b$ and $5 c$ with regards to the purpose of bone grafting).

The patient was assessed for fitness at the anaesthetic clinic and was found to be fit and healthy without any medical problem or allergies 1 week prior to the orthognathic surgery and following admission. The orthognathic surgery involved a Le Fort I maxillary advancement and a BSSO for the setback of the mandible. The maxillary third molars were removed at the time of the surgery to facilitate the tuberosity osteotomy bilaterally. Fixation was achieved using two Lshaped miniplates on either side for the maxilla at the piriform rim and the zygomatic buttress bilaterally. One 4-hole mini plate (MatrixORTHOGNATHIC, Pennsylvania, United States) was fixed on the upper end of the proximal and distal segments of the mandible after the mandibular sagittal split osteotomy. Mandibular cortico-cancellous bone that was removed from the proximal segment after the sagittal split osteotomy was grafted to the labial cortical alveolar area where there was a defect using $2 \times 1.5 \mathrm{~mm}$ miniscrews (MatrixORTHOGNATHIC, Pennsylvania, United States) (Figure 4b). After the bimaxillary surgery, the patient underwent a period of postsurgical orthodontics to tidy up the final occlusion. This took a further 19 months to complete due to the patient's busy schedule.

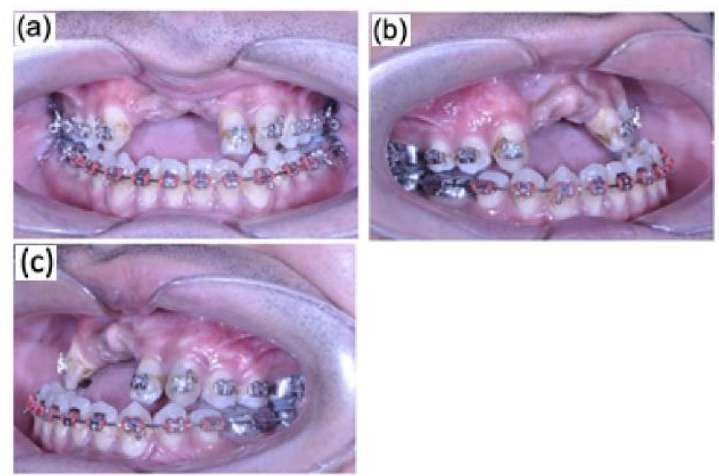

Figure 5: Pre-surgical orthodontic treatment with fixed appliance

Rehabilitation with implants after the completion of the post-surgical orthodontics was relatively straightforward using a conformative approach. However, remodelling of the grafted corticocancellous bone especially at tooth 12 region was evident in the CBCT (Figure 4d) at the time of implant planning. It was decided to concurrently perform guided bone regeneration (GBR) at the first stage implant surgery. A trapezoidal flap was raised and the 4 mini-screws that held the corticocancellous graft were removed. Following this, two $3.5 \mathrm{~mm} \times 10 \mathrm{~mm}$ AnyRidge implants (MEGAGEN, Seoul, Korea) were placed at the site of teeth 12 and 21 after standard implant osteotomy preparation (Figure 6a). The alveolar bone overlying the implants were layered with freeze dried cortical bone allograft (Sure-Oss, Pennsylvania, United States) and covered with a $15 \times 20 \mathrm{~mm}$ resorbable 
collagen membrane (Dentium, Gyeonggi-do, Korea) prior to flap closure. Second stage surgery took place eight months post-implant placement, whereby healing abutments were placed for two weeks to allow for soft-tissue healing before making a pick-up impression using a polyether impression material in an open-tray (Figure 6b). Porcelain fused to metal bridge was fabricated and cemented in place using temporary cement (TempBond $^{\mathrm{TM}}$, California, United States) on a 25을 angled abutment at the site of tooth 12 and a straight abutment at the site of tooth 21 (Figures $6 e$ and $6 f$ ).
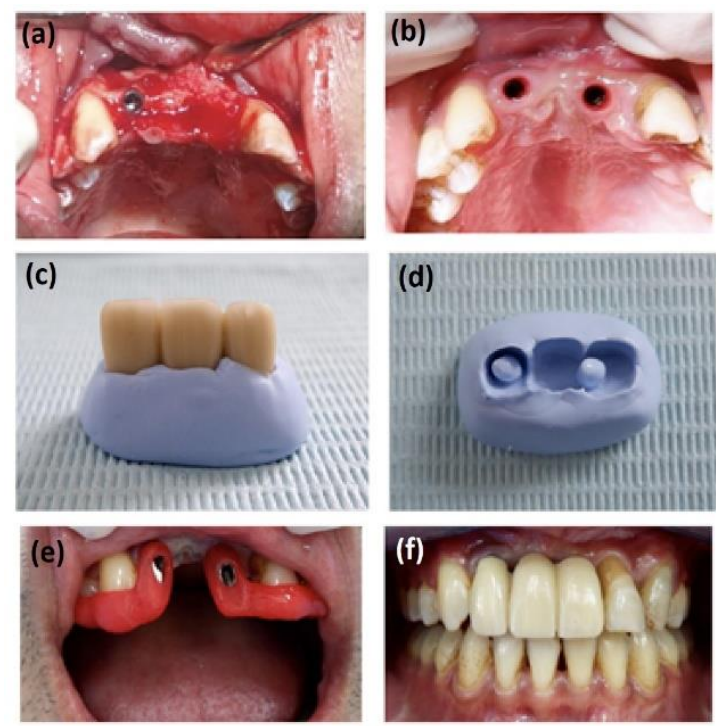

Figure 6: (a) First-stage implant surgery, (b) Two weeks following second-stage surgery, (c)\&(d) cementation device, (e) Insertion of angled abutments using a pattern resin jig and ( $f$ ) Cemented fixed prosthesis

At six months review, a composite build-up was done on tooth 22 to improve its appearance by masking the root that had undergone recession of the gingiva (Figure 6f). Overall, the patient was highly satisfied with the appearance and was able to function well with the prosthesis (Figure 7).

\section{DISCUSSION}

This case highlights the approach to the management of a patient who had experienced avulsed anterior maxillary teeth during childhood which required implant rehabilitation. Despite having been through the oral health system since the age of 11 years old, the patient received treatment that only focused on the TDI such as the replacement of missing teeth and endodontic therapy of non-vital teeth without a holistic approach. An early intervention aimed at expansion and protraction of maxilla to correct the anterior crossbite with a functional appliance in the pre- pubertal period may have prevented the need for surgical correction. ${ }^{3}$
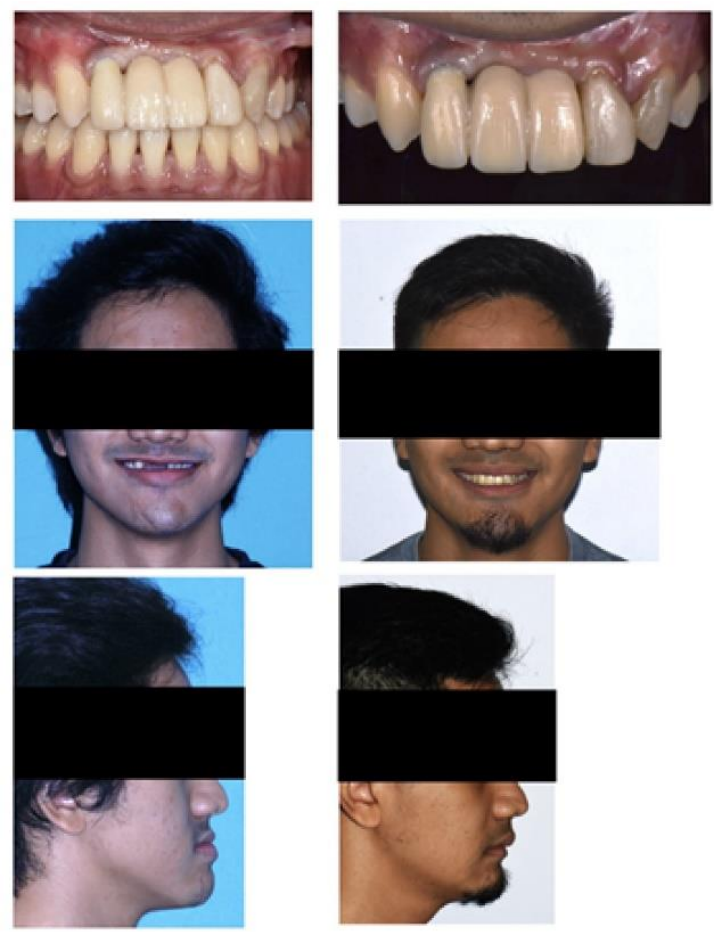

Figure 7: Post-treatment intraoral and extraoral photographs

Also, should the implants have been placed without the correction of the jaw discrepancies, orthodontic decompensation of the implants which are osteointegrated would not have been possible. In the event that bimaxillary surgery is still performed with implants in place, the surgical result would be compromised by the proclination of the implant prosthesis. Thus, comprehensive planning, timing and sequence of treatment plays a great importance in minimizing treatment complexity for a patient who had suffered from TDI with a Class III dentoalveolar discrepancies.

In deciding whether surgery is indicated in nongrowing patients, the literature has pointed to some cephalometric indicators. It has been suggested that ANB angle of $-4^{0}$, lower incisor to mandibular plane angle inclination of $83^{\circ}$ and Holdaway angle of $3.5^{\circ}$ are threshold values for the possibility of correction with an orthodontic appliance. $^{4}$ Recent retrospective investigation reported greater Holdaway angle whereby surgery was suggested for Holdaway angle of less than $10.3^{0}$. Apart from this, the investigation also mentioned that a Wit's appraisal of less than -5.8 $\mathrm{mm}$ must be treated by surgery. ${ }^{5}$ With an ANB angle of $-1^{0}$, lower incisor to mandibular plane angle inclination of $85^{\circ}$, and a Wits appraisal of $-17 \mathrm{~mm}$, the patient falls into the combined surgery and orthodontic appliance category. 
The 1 to 5 years post-surgical relapse of this patient is expected to be none or minimal. It has been reported that the maxillary advancement and mandibular setback with rigid fixation is a relatively stable procedure, with moderate relapse of $2 \mathrm{~mm}$ to $4 \mathrm{~mm}$ for maxillary advancement occurring in $20 \%$ of patients in the first year with minimal changes after that while any relapse in the mandibular setback post-surgery can be due to the musculature returning the mandibular ramus to the original position. This has been overcome with improved surgical technique over the years. ${ }^{6}$

The patient's overall treatment went according to plan except for the difficulty encountered in achieving the adequate labial bone volume in the anterior maxilla for implants placement. A staged approach of bone grafting followed by implant placement is the clinical norm when there is bone insufficiency for implant placement. In this case, the initial bone grafting that was performed during the orthognathic surgery was to avoid an additional surgical procedure later for the patient and also to use the cortico-cancellous that was readily available from the mandible. Autogenous bone block graft is considered the gold standard in overcoming alveolar ridge deficiency compared to GBR with more significant osteogenic inductive potential and less volume resorption of around $7.2 \%$ for the former as compared to around $12.5 \%$ for GBR. ${ }^{7}$ However, the prolonged time interval between the initial bone grafting at the anterior maxilla and the placement of implants resulted in bone remodelling/bone loss. This necessitated the second bone grafting procedure at the time of the implant first stage surgery.

The implants were restored using a cementretained i-FDP. The advantage of having a cementretained restoration is that the screw access of the implant abutment towards the incisal or labial aspect is covered by the prosthesis that is cemented. In addition to this, it is easier to achieve passivity of the bridge at the prosthesis-abutment level rather than at the abutment-implant connection level. A systematic review of cemented and screw-retained i-FDP has revealed no difference in terms of 5-year survival rate between the two types of prosthesis but with less technical and biological complications favouring the screwretained prosthesis. ${ }^{8}$

The main disadvantages of a cemented prosthesis are the excess cement may lead to peri-implantitis and a problem with retrievability should there be a need to remove the bridge in the long run. In order to overcome these possible complications, the bridge was cemented using a temporary cement Ann Dent UM. 2021, 28: 8-14 with an aid of a cementation device made using polyvinylsiloxane putty material (Aquasil, Pennsylvania, United States). The usage of this device has been reported and, it is mainly to displace the excess cement from the intaglio of the i-FDP prior to the final cementation to minimize the extent of set cement around the implant abutments and the peri-implant soft tissue (Figures $6 \mathrm{c}$ and 6d)..$^{9,10}$ Recently, it has been reported that the retrievability of i-FDP is related to the height and taper of the abutments and the type of luting cement used. The best retrievability was obtained with long, slightly tapered abutments and when the prosthesis is cemented with temporary luting agent. $^{11}$

A choice of ceramic veneer or direct composite build-up was discussed with the patient for masking the root of 22 due to the gingival recession. The gingival recession could have been caused by a combination of factors which include labial bone dehiscence related to orthodontic movement of teeth with the fixed appliance and the surgical flap manipulation during the first stage implant surgery. The patient opted for the composite build-up due to the quick and minimal invasiveness of the procedure and the good prognosis of this treatment option. ${ }^{12}$ This case would have been more challenging to restore if the patient was to display the cervical portion of his teeth when smiling. The low smile has been described as a smile that reveals less than $75 \%$ of the maxillary anterior teeth as seen in this patient. The advantage of the low smile line resulted in an acceptable aesthetics of the i-FDP without having to incorporate pink porcelain at the cervical margin of the prosthesis to camouflage the increased tooth length. ${ }^{13}$

Future consideration for this patient is to redirect the screw-access of the implants using angled abutments also known as dynamic abutments that comprise of castable cobalt-chromium or nickelchromium titanium base that allows for deviation of the implant screw access up to 28 degrees in order to attain a screw retained prosthesis. ${ }^{14}$ However, with good home care and routine follow-up, prognosis of the cement retained i-FDP and the overall treatment given to the patient is expected to be favourable.

\section{CONCLUSION}

Prosthodontic rehabilitation of missing maxillary anterior teeth with implants in a patient with normal maxillary and mandibular relations is a straight forward task. But in a patient with a severe Class III malocclusion, the correction of the jaw deformity takes precedence before implant 
placement. Any attempt to place implants in this patient without the correction of the jaw deformity will result in poor aesthetic, function and an unhappy patient.

\section{ACKNOWLEDGEMENT}

The authors would like to thank the patient for his co-operation throughout the treatment period. Our appreciation goes to the Dean and staff of Faculty of Dentistry, Universiti Teknologi MARA for their ever continuing support. Special thanks are given to the Director General of Malaysia, Principle Director of Oral Health Division and all members of staff of the Oral Health Division, Negeri Sembilan, Ministry of Health, Malaysia who were directly or indirectly involved in the management of this patient.

\section{DECLARATION OF INTEREST}

All authors deny any conflict of interest in the management and reporting of this case.

\section{SOURCE OF FUNDING}

This patient was managed as part of the main author's employment at the Faculty of Dentistry, Universiti Teknologi MARA (UITM) and co-author's previous employment at Hospital Tuanku Jaafar, Negeri Sembilan, Ministry of Health, Malaysia without any external funding.

\section{REFERENCES}

1. Azami-Aghdash S, Azar EF, Azar PF, Rezapour A, Moradi-Joo M, Moosavi A, Oskouei GS. Prevalence, aetiology, and types of dental trauma in children and adolescents: systematic review and meta-analysis. Med J Islam Repub Iran. 2015;29:234.

2. Petti S, Glendor U, Andersson L. World traumatic dental injury prevalence and incidence, a meta-analysisone billion living people have had traumatic dental injuries. Dent Traumatol. 2018;34:71-86.

3. Oltramari-Navarro PV, de Almeida RR, Conti AC, Navarro Rde L, de Almeida MR, Fernandes LS. Early treatment protocol for skeletal Class III malocclusion. Braz Dent J. 2013;24(2):167-173.

4. Kerr WJ, Miller S, Dawber JE. Class III malocclusion: surgery or orthodontics? Br J Orthod. 1992;19(1):21-24.

5. Eslami S, Faber J, Fateh A, Sheikholaemmeh F, Grassia V, Jamilian A. Treatment decision in adult patients with class III malocclusion: surgery versus orthodontics. Progress in orthodontics. 2018;19:28.

6. Proffit WR, Turvey TA, Phillips $C$. The hierarchy of stability and predictability in orthognathic surgery with rigid fixation: an update and extension. Head Face Med. 2007;3:21.

7. Gultekin BA, Bedeloglu E, Kose TE, Mijiritsky E. Comparison of Bone Resorption Rates after Intraoral Block Bone and Guided Bone Regeneration Augmentation for the Reconstruction of Horizontally Deficient Maxillary Alveolar Ridges. Biomed Res Int. 2016;2016:4987437.

8. Wittneben JG, Millen C, Brägger U. Clinical performance of screw- versus cement-retained fixed implantsupported reconstructions--a systematic review. Int J Oral Maxillofac Implants. 2014;29 Suppl:84-98.

9. Chee WW, Duncan J, Afshar M, Moshaverinia A. Evaluation of the amount of excess cement around the margins of cement-retained dental implant restorations: the effect of the cement application method. J Prosthet Dent. 2013;109(4):216-221.

10. Bukhari SA, AlHelal A, Kattadiyil MT, Wadhwani C, Taleb A, Dehom S. An in vitro investigation comparing methods of minimizing excess luting agent for cement-retained implant-supported fixed partial dentures. J Prosthet Dent. 2020;124(6), 706-715.

11. Lugas AT, Terzini M, Zanetti EM, Schierano G, Manzella C, Baldi D, Bignardi C, Audenino AL. In Vitro Simulation of Dental Implant Bridges Removal: Influence of Luting Agent and Abutments Geometry on Retrievability. Materials (Basel). 2020 Jun 21;13(12):2797.

12. Demirci M, Tuncer S, Öztaş E, Tekçe N, Uysal Ö. A 4-year clinical evaluation of direct composite build-ups for space closure after orthodontic treatment. Clin Oral Investig. 2015;19(9):2187-2199.

13. Tjan AH, Miller GD, The JG. Some esthetic factors in a smile. J Prosthet Dent. 1984;51:24-8.

14. Gómez-Polo M, Gómez-Polo C, Celemín A, Ortega R. Interim restoration using dynamic abutments to re-treat a single-implant crown with a labial angulation: A clinical report. J Prosthet Dent. 2018;120(6):791-795. 


\section{Editorial History}

Date of Submission: 13 Nov 2020

Review \& Revision: 20 Nov 2020 - 15 Jan 2021

Accepted: 15 Jan 2021

Published:

License Information: This work is licensed under a Creative Commons Attribution 